\title{
Highly Sensitive Temperature Sensor Based on Isopropanol-Filled Photonic Crystal Fiber Long Period Grating
}

\author{
Chao Du', Qi Wang ${ }^{1,2, *}$, Yong Zhao ${ }^{1,2}$, Jin $\mathrm{Li}^{1}$ \\ ${ }^{1}$ College of Information Science and Engineering, Northeastern University, Shenyang 110819, China \\ ${ }^{2}$ State Key Laboratory of Synthetical Automation for Process Industries (Northeastern University), Shenyang 110819, \\ China \\ *Corresponding author Email: wangqi@ise.neu.edu.cn
}

\section{Corresponding Author: Qi Wang}

Qi Wang is with College of Information Science and Engineering, Northeastern University, and State Key Laboratory of Synthetical Automation for Process Industries (Northeastern University).

Email: wangqi@ise.neu.edu.cn

Telephone number: $+86-13889195280$

\section{Mailing address:}

Northeastern University, College of Information Science and Engineering

P. O. Box 321, Shenyang, Liaoning, 110819, China

Chao Du and Jin Li are with College of Information Science and Engineering, Northeastern University, Shenyang, 110819, China.

\section{Mailing address:}

Northeastern University, College of Information Science and Engineering

P. O. Box 321, Shenyang, Liaoning, 110819, China

Yong Zhao is with College of Information Science and Engineering, Northeastern University, and State Key Laboratory of Synthetical Automation for Process Industries (Northeastern University).

\section{Mailing address:}

Northeastern University, College of Information Science and Engineering

P. O. Box 321, Shenyang, Liaoning, 110819, China 


\title{
Highly Sensitive Temperature Sensor Based on Isopropanol-Filled Photonic Crystal Fiber Long Period Grating
}

\author{
Chao Du1, Qi Wang ${ }^{1,2, *}$, Yong Zhao ${ }^{1,2}, \mathrm{Jin} \mathrm{Li}^{1}$ \\ ${ }^{1}$ College of Information Science and Engineering, Northeastern University, Shenyang 110819, China \\ ${ }^{2}$ State Key Laboratory of Synthetical Automation for Process Industries (Northeastern University), Shenyang 110819, \\ China \\ *Corresponding author Email: wangqi@ise.neu.edu.cn
}

\begin{abstract}
A high sensitivity measurement method for temperature has been proposed and investigated based on an isopropanol-filled photonic crystal fiber long period grating (PCF-LPG). Due to the high thermo-optic coefficient (TOC) of isopropanol, the sensitivity of the proposed temperature sensor could be effectively improved by filling isopropanol in the air waveguides of PCF. It can be found that the resonant dip will be split in two dips after filling isopropanol and the two dips have different sensitivities to surrounding temperature. Because of PCF-LPG is sensitive to the refractive index (RI) of internal filled liquid, the isopropanol-filled PCF-LPG temperature sensor has a high sensitivities of $1.356 \mathrm{~nm} /{ }^{\circ} \mathrm{C}$ in the range of $20^{\circ} \mathrm{C}$ to $50^{\circ} \mathrm{C}$. The simplicity and the excellent performance of our proposed device make it potential for the applications of high-precision temperature measurement is required.
\end{abstract}

Keywords: Photonic crystal fiber; Long period grating; Optical fiber sensors; Temperature measurement; High sensitivity.

\section{Introduction}

As a key physical parameter, temperature is closely related to the characteristics of material. The measurement of temperature has great significance to the fields where high-precision temperature measurement is required, especially in food industries or chemical for quality control and biochemical reactions monitoring ${ }^{[1]}$. Compared to other temperature sensors, optical temperature sensors have many advantages such as compact structure, fast response, high sensitivity, high stability, resistance to electromagnetic interference ${ }^{[2]}$. In recent years, various types of optical temperature sensors have been widely studied, such as interferometer structures ${ }^{[3-8]}$, fiber loop structures ${ }^{[9-10]}$, fiber grating structures ${ }^{[11-13]}$, etc. The measurement mechanisms of most devices mainly depend on the thermo-optic properties of silica. Due to a low thermo-optic coefficient (TOC) of pure silica, the temperature sensitivities of them are relatively low. In order to enhance the sensitivities of optical fiber temperature sensors, there have been many reports about the combinations of optical fiber sensors and materials with a high TOC ${ }^{[14-15]}$. The thermo-optic effect and 
thermal expansion effect of sealed liquid can turn it into an ultrasensitive temperature sensor, because of the microfiber taper is highly sensitive to the surrounding RI. Although the temperature sensitivity is as high as $3 \mathrm{~nm} /{ }^{\circ} \mathrm{C}$, it is easy to be broken due to the diameter of the taper is only about $10 \mu \mathrm{m}$.

With the emergence and development of PCF, temperature sensors based on PCF structures have been widely reported. 2011, Wang et al. proposed a selectively infiltrated PCF with ultrahigh temperature sensitivity, the average temperature sensitivity achieved is $54.3 \mathrm{~nm} /{ }^{\circ} \mathrm{C}{ }^{[16]}$. Such a device is demonstrated in their experiment by filling standard $1.46 \mathrm{RI}$ liquid into one of the air holes of the commercially available PCF by use of femtosecond laser-assisted complicated technique. The disadvantage of these selectively infiltrated PCF temperature sensors is that the fabrication process is complicated and expensive. 2012, Qian et al. proposed a high-sensitivity temperature sensor based on an alcohol filled PCF loop mirror, and experimental results demonstrated that the temperature sensitivity can reach up to $6.6 \mathrm{~nm} /{ }^{\circ} \mathrm{C}{ }^{[17]}$. Due to complicated sensing structure, the loop structure measurement systems always show poor stability.

The material with a high TOC will produce a large RI change with surrounding temperature variation, so the measurement of temperature can be achieved by monitoring RI change. LPG written in PCF can couple the core mode to the forward propagating cladding modes, and shows relatively high RI resolution of $10^{-5}$ $10^{-7}$ RIU because of the strong interaction between cladding modes and filled medium ${ }^{[18-19]}$, so it can be used for the measurement of temperature. Isopropanol is a transparent liquid with a high TOC and its RI is lower than that of the silica core region ${ }^{[20]}$, which is easy to be integrated with PCF. In this letter, a highly sensitive temperature sensor based on an isopropanol-filled PCF-LPG has been proposed and demonstrated. The results indicated that the sensitivity of the proposed temperature sensor can reach $1.356 \mathrm{~nm} /{ }^{\circ} \mathrm{C}$ with a LPG length of $2.7 \mathrm{~cm}$ and a grating period of $180 \mu \mathrm{m}$, in the range of $20^{\circ} \mathrm{C}$ to $50^{\circ} \mathrm{C}$. It has a high measurement resolution of $0.015^{\circ} \mathrm{C}$ and advantages of easy fabrication, compact structure, and good linearity.

\section{Sensing Structure and Measurement Mechanism}

The schematic diagrams of the isopropanol-filled PCF-LPG sensing system are shown in Fig. 1. A broadband light source (ASE) and an optical spectrum analyzer (OSA) with a wavelength resolution of $20 \mathrm{pm}$ were adopted to measure the transmission spectra of our proposed device. In actual measurements, the sensing probe should be placed in a temperature-controlled chamber and fixed with two fiber holders to keep it straight. 
ASE Temperature-controlled chamber

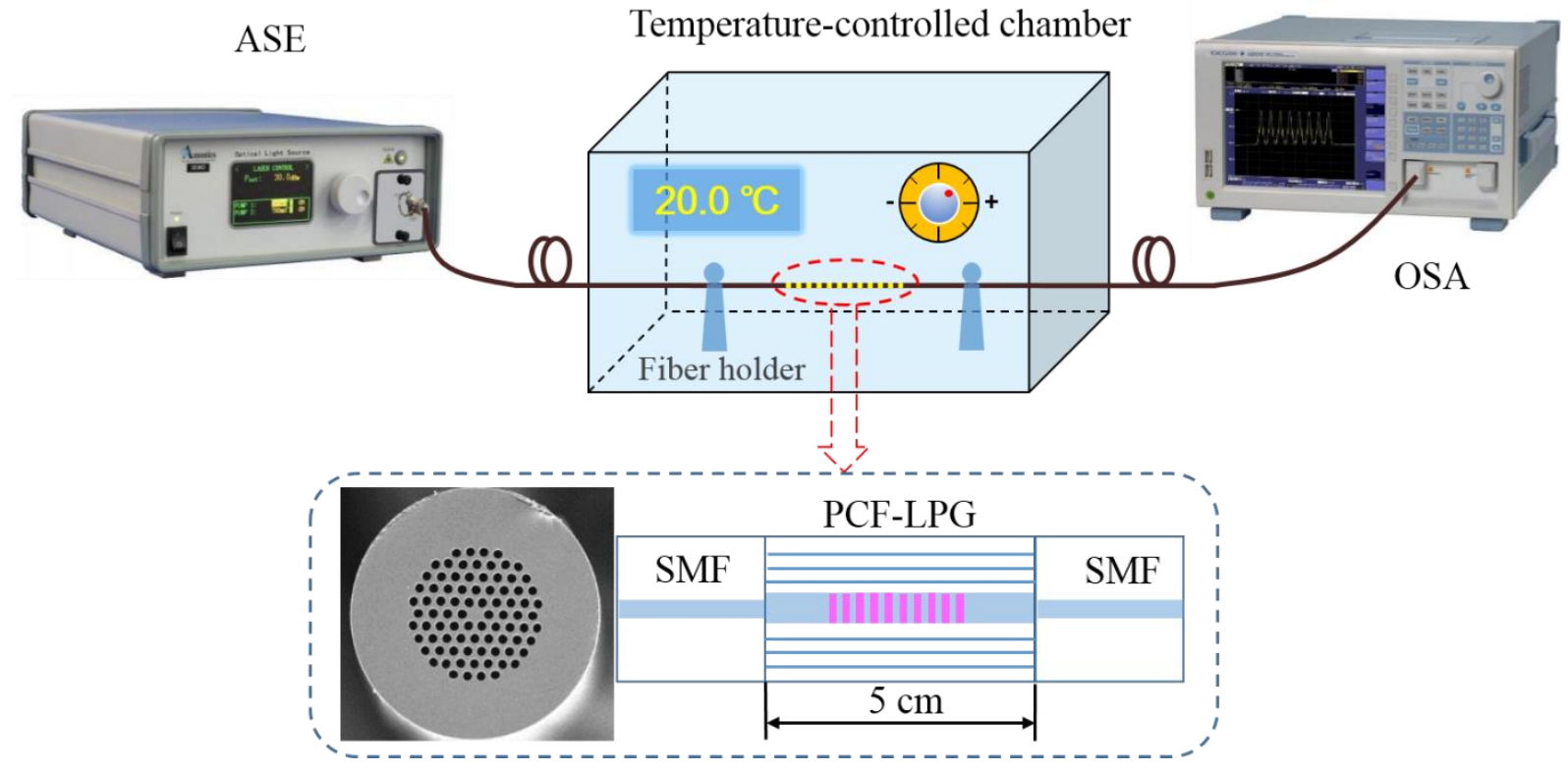

Fig. 1. Schematic diagrams of the isopropanol-filled PCF-LPG sensing system.

Inset is the enlarged structure of temperature sensor.

As shown in Fig. 1, the adopted PCF has a hole-diameter of $4.88 \mu \mathrm{m}$ and a hole-spacing of $7.67 \mu \mathrm{m}$. In general, the air waveguides of PCF can be filled with isopropanol by capillary action. The length of PCF was set to $5 \mathrm{~cm}$, which ensured the LPG was completely immersed in isopropanol. Finally, the isopropanol-filled PCF-LPG temperature sensor was obtained after splicing with SMFs by using a commercial fiber splicer.

The LPG can couple the core mode to the forward propagating cladding modes. The coupling between the core mode and the $m$ th cladding mode matches the phase-matching conditions:

$$
\beta_{c o}-\beta_{c l, m}=2 \pi / \Lambda
$$

where $\beta_{c o}$ and $\beta_{c l, m}$ are the propagation constants of the core mode and the $m$ th order cladding mode, respectively. $\Lambda$ is the grating period. Therefore, resonant wavelength $\lambda_{\text {res }}$ is determined by:

$$
\lambda_{\text {res }}=\left(n_{e f f}^{c o}-n_{e f f}^{c l, m}\right) \Lambda=\Delta_{e f f} \Lambda
$$

where $n_{e f f}^{c o}$ and $n_{e f f}^{c l, m}$ are the effective refractive indices of core mode and the $m$ th cladding mode, respectively.

The proposed air-filled PCF-LPG with a grating period of $180 \mu \mathrm{m}$ and a length of $2.7 \mathrm{~cm}$, and then recorded its transmission spectrum at room temperature, which is shown in Fig. 2. The resonant dip was induced by the coupling of core mode $\mathrm{LP}_{01}$ and cladding mode LP02 (with a resonant wavelength of 1502.5 $\mathrm{nm})$. 


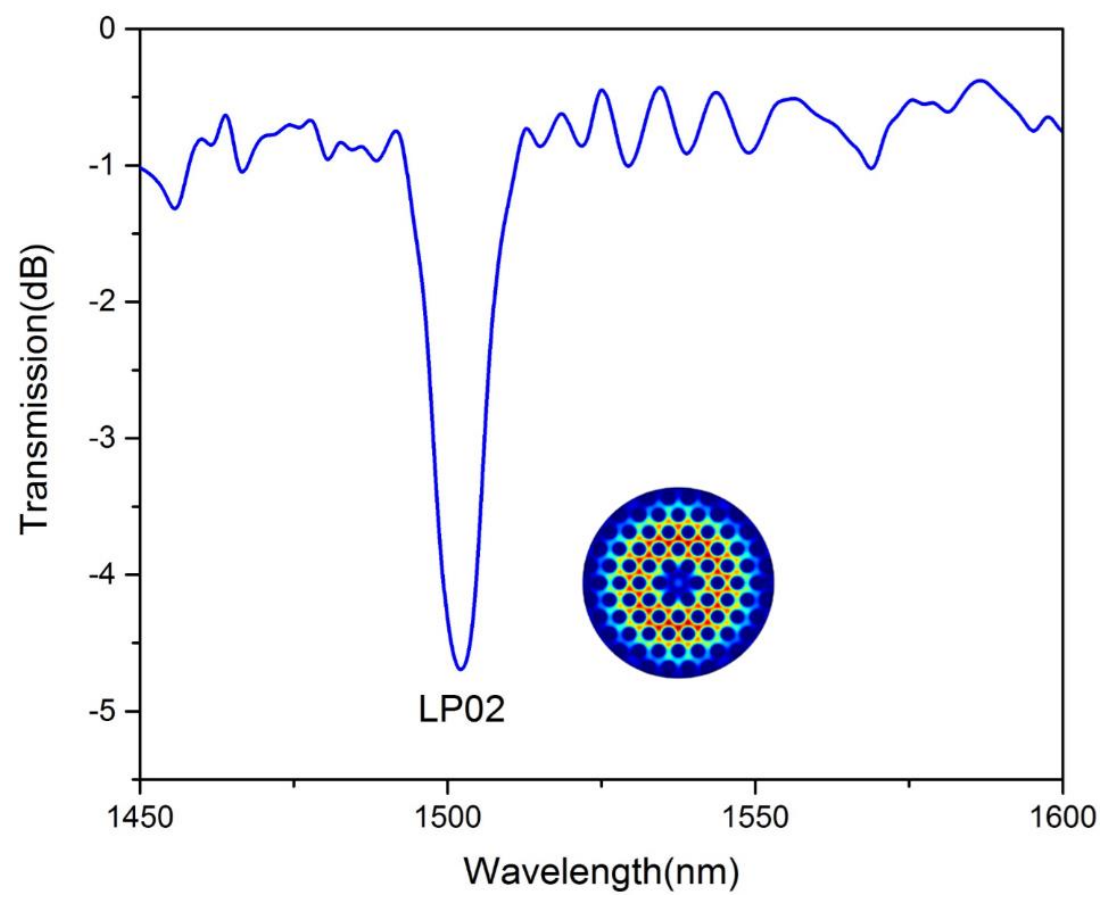

Fig. 2. Transmission spectrum of air-filled PCF-LPG at room temperature.

The sensitivity of LPG temperature sensor is mainly determined by the thermal expansion and thermooptic effects of silica. Considering the influence of temperature, the phase-matching conditions is given by:

$$
\beta_{c o}(\lambda, T)-\beta_{c l, m}(\lambda, T)=2 \pi / \Lambda
$$

where $\beta_{c o}(\lambda, T)$ and $\beta_{c 1, m}(\lambda, T)$ are the propagation constants of core mode and the $m$ th order cladding mode when temperature is $T$ and resonant wavelength is $\lambda . \Lambda$ is the grating period when temperature is $T$, as shown in Eq. (4).

$$
\Lambda=(1+\alpha \Delta T) \Lambda_{0}
$$

Where $\alpha$ represents thermal expansion coefficient (TEC). The temperature sensitivity $K_{T}$ is given by [12]:

$$
K_{T}=\frac{d \lambda_{\text {res }}}{d T}=\left(\frac{\partial n_{e f f}^{c o}}{\partial T}-\frac{\partial n_{e f f}^{c l, m}}{\partial T}\right) \Lambda+\left(n_{e f f}^{c o}-n_{e f f}^{c l, m}\right) \frac{\partial \Lambda}{\partial T}
$$

As shown in Eq. (5), the thermal expansion effect changes the grating period and the thermo-optic effect changes the effective refractive indices of core mode and cladding modes. The thermo-optic effect is the dominant factor of temperature sensitivity because of the TOC $\left(8.6 \times 10^{-6} /{ }^{\circ} \mathrm{C}\right)$ is an order of magnitude higher than $\operatorname{TEC}\left(5.5 \times 10^{-7} /^{\circ} \mathrm{C}\right)$.

As shown in Fig. 3, the temperature sensing characteristics of air-filled PCF-LPG have been investigated by heating up from $20{ }^{\circ} \mathrm{C}$ to $50{ }^{\circ} \mathrm{C}$ in steps of $10{ }^{\circ} \mathrm{C}$, and the red-shifts of resonant dip are observed with temperature increase. The resonant wavelength shifts at different temperature are shown in Fig. 4. The resonant dip shifts toward a longer wavelength direction, which are consistent with the temperature sensitivity characteristics of LPG. The fitting curves are also presented in Fig. 4, in which the temperature 
sensitivity for air-filled PCF-LPG is only $9.1 \mathrm{pm} /{ }^{\circ} \mathrm{C}$ due to the lower TOC and TEC of silica.

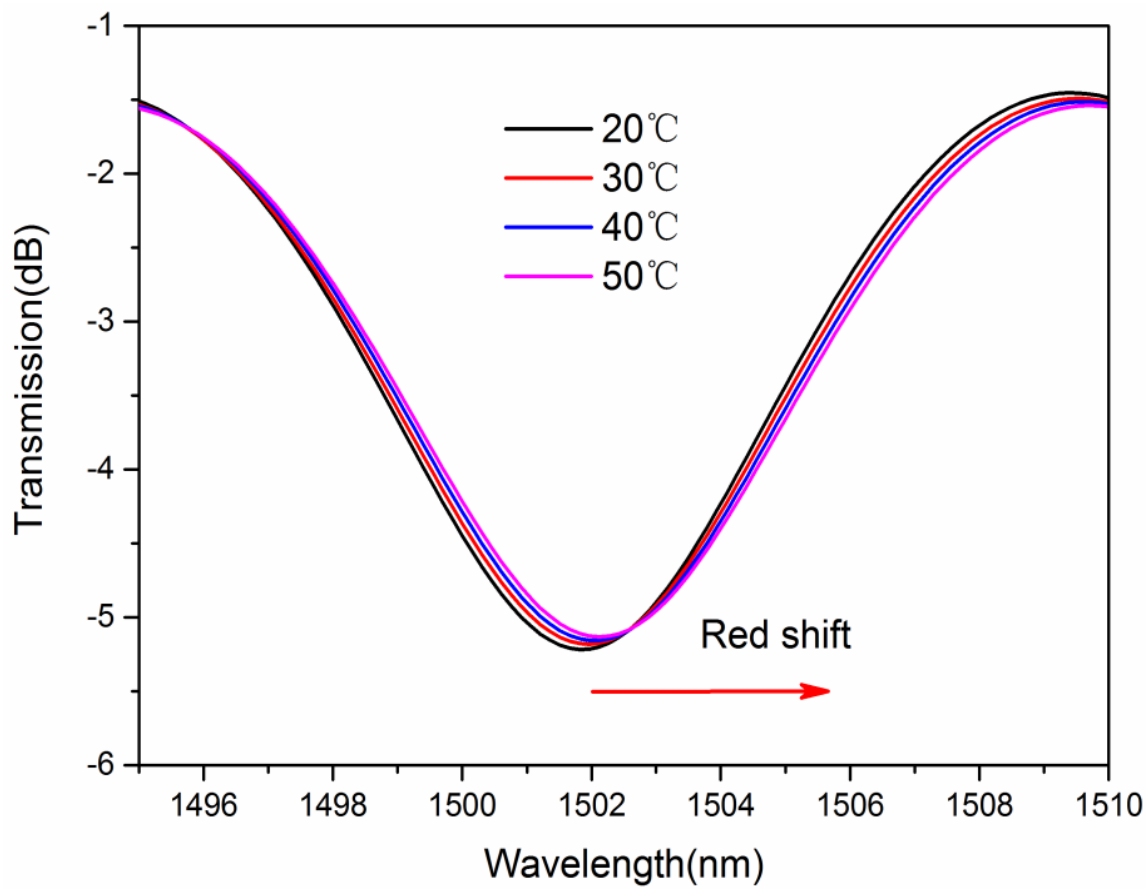

Fig. 3. Transmission spectrum of the air-filled PCF-LPG changes at different temperature.

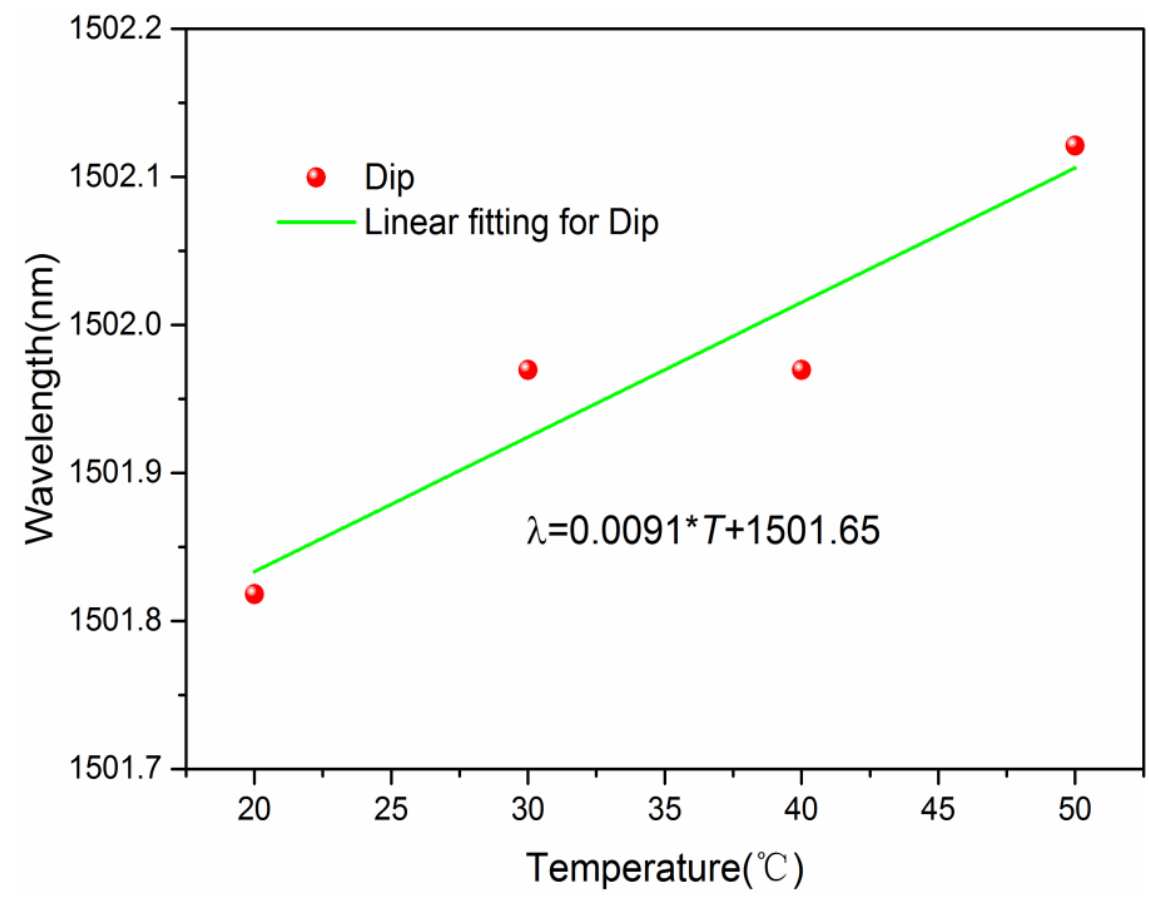

Fig. 4. Relationship between temperature and wavelength shift of resonant dip.

As mentioned above, the temperature sensitivity of air-filled PCF-LPG is as low as $9.1 \mathrm{pm} /{ }^{\circ} \mathrm{C}$ which can hardly meet the requirement of high-precision temperature measurement. Because PCF-LPG is sensitive to the RI of internal filled liquid, if the air waveguides of PCF are filled with a high TOC material, a small temperature change can induce a prominent shift of the resonant dip. Therefore, we can propose a highly sensitive temperature sensor based on an isopropanol-filled PCF-LPG. The measurement mechanism of the proposed device is to transfer the temperature response into the RI response of internal filled isopropanol.

\section{Results and Discussions}


The isopropanol-filled PCF-LPG was connected to ASE and OSA by using two commercial SMF adapters. Fig. 5 shows the transmission spectra of the device at room temperature $\left(20^{\circ} \mathrm{C}\right)$. The RI of isopropanol is about 1.37 which is higher than that of air $(\mathrm{RI}=1)$, so the transmission spectra of isopropanolfilled PCF-LPG is different from that of air-filled PCF-LPG. It can be found that the resonant dip will be split in two dips after filling isopropanol, due to the phase matching conditions of the interference are changed by the RI of internal filled materials. The splitting of the resonant peak is mainly caused by the birefringence in PCF-LPG ${ }^{[19]}$. The core mode has different effective indices corresponding the two orthogonal polarization modes because of the birefringence of PCF. The increased RI of isopropanol can enhance the birefringence of PCF, so the resonance split into two dips.

In order to investigate the temperature sensing characteristics of isopropanol-filled PCF-LPG. The temperature sensor was placed into a temperature-controlled chamber with a temperature resolution of $0.1{ }^{\circ} \mathrm{C}$, and then heated up from $20{ }^{\circ} \mathrm{C}$ to $50{ }^{\circ} \mathrm{C}$ in steps of $5{ }^{\circ} \mathrm{C}$. At each step, the temperature was kept for 10 minutes and the transmission spectrum was recorded after it stabled. As shown in Fig. 6, the blue-shifts of resonant dips are observed with temperature increase. The presence of isopropanol filled in the cladding air channels weakens the restriction of guided mode in core area. The intensity profile of this guide mode extends to the cladding region, and their resonance strengths decrease when isopropanol RI increases as coupling intensity is proportional to $\Delta_{\text {eff }}$ that decreases with increased RI in the air channels of PCF-LPG ${ }^{[19]}$. As a result, the resonance strengths increase with the increase of temperature due to the negative TOC of isopropanol.

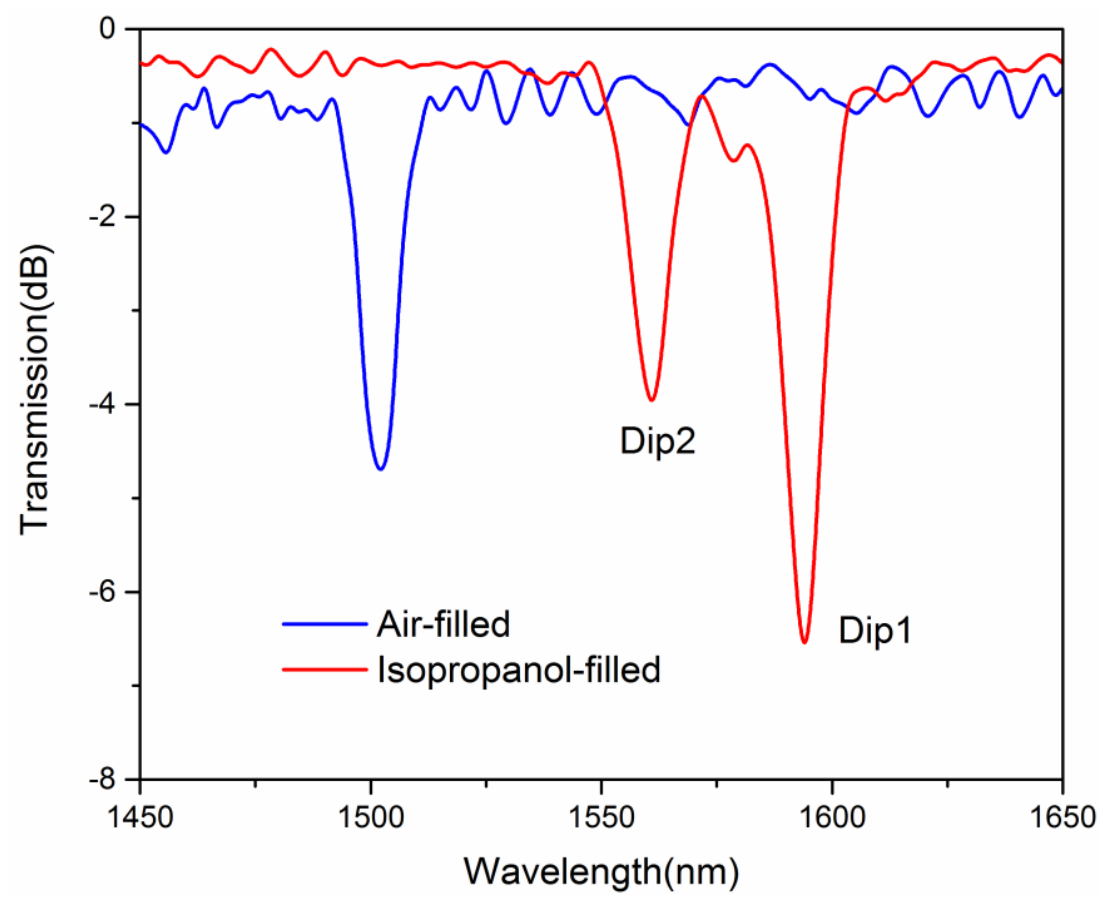

Fig. 5. Transmission spectra of the device when air waveguides were filled with air or isopropanol at room temperature $\left(20^{\circ} \mathrm{C}\right)$. 


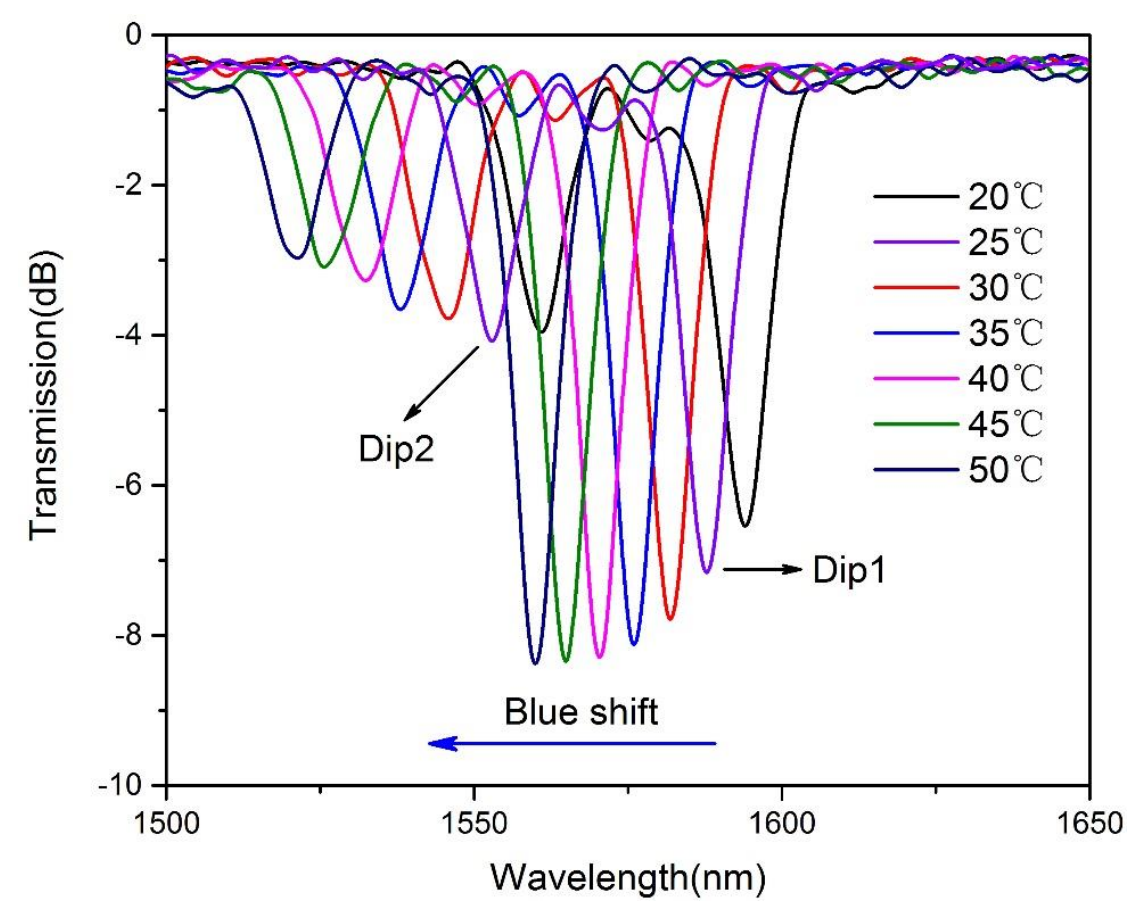

Fig. 6. Transmission spectrum of the isopropanol-filled PCF-LPG changes at different temperature.

The resonant wavelength shifts of two dips at different temperature are shown in Fig. 7. The resonant dips shift toward a shorter wavelength direction due to isopropanol exhibits a highly negative TOC $\left(-4.5 \times 10^{-}\right.$ ${ }^{4} /{ }^{\circ} \mathrm{C}$ ), which are consistent with the RI sensitivity characteristics of PCF-LPG. The fitting curves are also presented in Fig. 7, in which the temperature sensitivities for Dip1 and Dip2 are $1.356 \mathrm{~nm} /{ }^{\circ} \mathrm{C}$ and $1.124 \mathrm{~nm} /{ }^{\circ} \mathrm{C}$, respectively. The response of the sensor keeps good linearity in the measured temperature range.

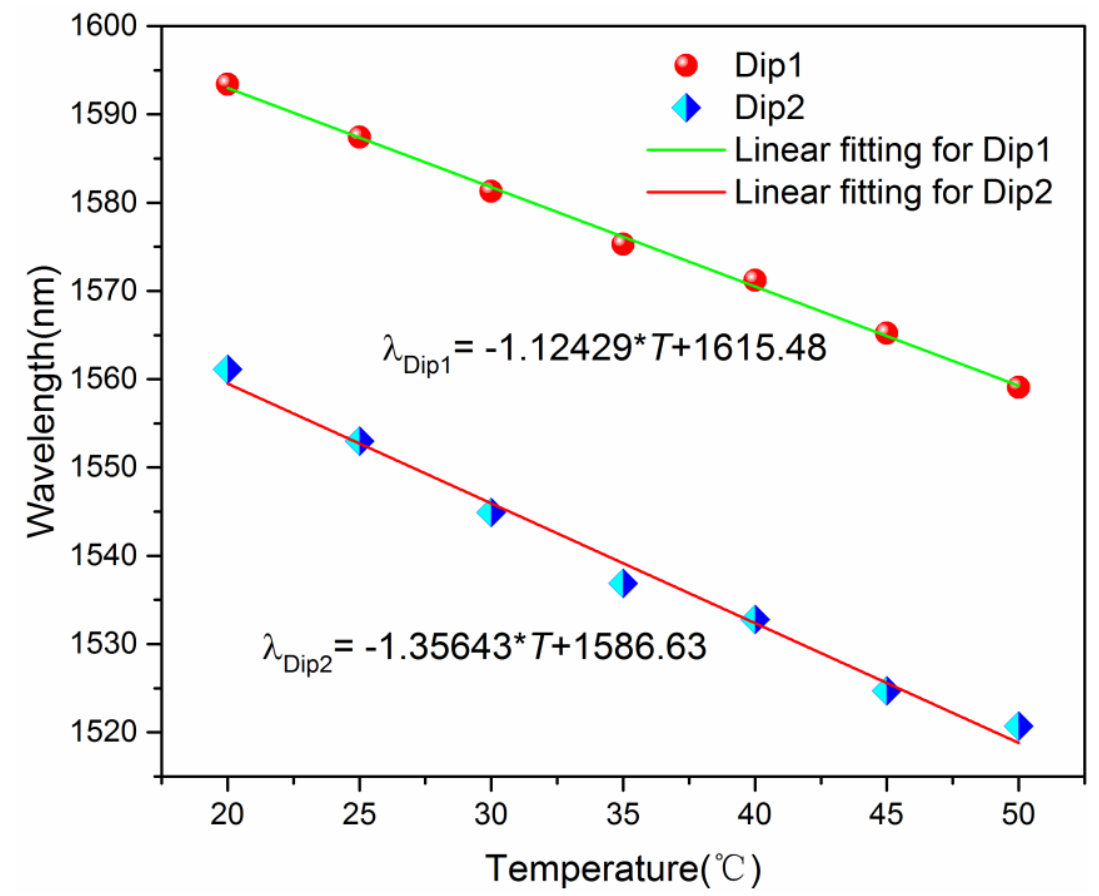

Fig. 7. Relationships between temperature and wavelength shift of Dip1 and Dip2, respectively.

In summary, the resonant dip will be split in two dips after filling isopropanol due to the enhancement of birefringence in PCF, and the two dips shows different temperature sensitivities. Within a temperature range of $20^{\circ} \mathrm{C}$ to $50^{\circ} \mathrm{C}$, the resonance wavelengths of the proposed sensor show linear temperature dependences. In 
order to obtain a relatively higher temperature sensitivity of $1.356 \mathrm{~nm} /{ }^{\circ} \mathrm{C}$, Dip2 can be selected for measuring temperature.

\section{Conclusion}

A novel method for the measurement of temperature with high sensitivity has been proposed and analyzed based on an isopropanol-filled PCF-LPG. The research results show that a high temperature sensitivity of $1.356 \mathrm{~nm} /{ }^{\circ} \mathrm{C}$ and a measurement resolution of $0.015^{\circ} \mathrm{C}$ are realized by filling isopropanol in the air waveguides of PCF. The proposed sensor with advantages of compact structure, high sensitivity and good linearity is promising to be used in practical applications based on high precision temperature measurement.

\section{Acknowledgement}

This work was supported by the Natural Science Foundation of Liaoning Province under Grant 201602262, the Fundamental Research Funds for the Central Universities under Grant N140405001 and N150401001, the National Science Foundation for Distinguished Young Scholars of China under Grant 61425003, the National Natural Science Foundation of China under Grant 51607028, and the State Key Laboratory of Synthetical Automation for Process Industries under Grant 2013ZCX09.

\section{References}

[1] P. Lu, L. Men, K. Sooley, and Q. Y. Chen. "Tapered fiber Mach-Zehnder interferometer for simultaneous measurement of refractive index and temperature," Appl. Phys. Lett., vol. 94, no. 13, pp. 131110, Mar. 2009.

[2] O. S. Wolfbeis, "Fiber-optic chemical sensors and biosensors," Anal. Chem., vol. 80, no. 12, pp. 4269-4283, May. $8,2008$.

[3] B. Sun et al., "Simultaneous measurement of pressure and temperature by employing Fabry-Perot interferometer based on pendant polymer droplet," Opt. Exp., vol. 23, no. 3, pp. 1906-1911, Feb. 2015.

[4] S. Zhang et al., "Temperature characteristics of silicon core optical fiber Fabry-Perot interferometer," Opt. Lett., vol. 40, no. 7, pp. 1362-1365, Apr. 2015.

[5] L. Jiang, J. Yang, S. Wang, B. Li, and M. Wang, "Fiber Mach-Zehnder interferometer based on microcavities for high-temperature sensing with high sensitivity," Opt. Lett., vol. 36, no. 19, pp. 3753-3755, Oct. 2011.

[6] D. Wu, T. Zhu, K. S. Chiang, and M. Deng, "All single-mode fiber Mach-Zehnder interferometer based on two peanut-shape structures," J. Lightw. Technol., vol. 30, no. 5, pp. 805-810, Mar. 1, 2012.

[7] R. Yang, Y. S. Yu, Y. Xue, C. Chen, Q.-D. Chen, and H.-B. Sun, "Single S-tapered fiber Mach-Zehnder interferometers," Opt. Lett., vol. 36, no. 23, pp. 4482-4484, Dec. 2011.

[8] D. Wu, T. Zhu, and M. Liu, "A high temperature sensor based on a peanut-shape structure Michelson interferometer," Opt. Commun., vol. 285, no. 24, pp. 5085-5088, Nov. 1, 2012. 
[9] A. N. Starodumov, L. A. Zenteno, D. Monzon, and E. De la Rosa, "Fiber Sagnac interferometer temperature sensor," Appl. Phys. Lett., vol. 70, no. 1, pp. 19-21, Jan. 1997.

[10] E. De la Rosa, L. A. Zenteno, A. N. Starodumov, and D. Monzon, "All-fiber absolute temperature sensor using an unbalanced high-birefringence Sagnac loop,” Opt. Lett., vol. 22, no. 7, pp. 481-483. Apr. 1997.

[11] C. Chen et al., "Monitoring thermal effect in femtosecond laser interaction with glass by fiber Bragg grating," $J$. Lightw. Technol., vol. 29, no. 14, pp. 2126-2130, Jul. 15, 2011.

[12] X. Shu, L. Zhang, and I. Bennion, "Sensitivity characteristics of long period fiber gratings," J. Lightw. Technol., vol. 20, no. 2, pp. 255-266, Feb. 2002.

[13] J. Luo et al., "A magnetic sensor based on a hybrid long-period fiber grating and a magnetic fluid," IEEE Photon. Technol. Lett., vol. 27, no. 9, pp. 998-1001, May. 2015.

[14] Y. Xue et al., "Ultrasensitive temperature sensor based on an isopropanol-sealed optical microfiber taper," Opt. Lett., vol. 38, no. 8, pp. 1209-1211, Apr. 2013.

[15] H. R. Iván, M. H. David, M. H. Carlos, M. H. David, and V. Joel, "Highly sensitive temperature sensor based on a polymer-coated microfiber interferometer," IEEE Photon. Technol. Lett., vol. 27, no. 24, pp. 2591-2594, Dec. 2015.

[16] Y. Wang et al., "Selectively infiltrated photonic crystal fiber with ultrahigh temperature sensitivity," IEEE Photon. Technol. Lett., vol. 23, no. 20, pp. 1520-1522, Oct. 2011.

[17] W. Qian et al., "High-sensitivity temperature sensor based on an alcohol filled photonic crystal fiber loop mirror," Opt. Lett., vol. 36, no. 9, pp. 1548-1550, May. 2012.

[18]L. Rindorf, O. Bang, "Highly sensitive refractometer with a photonic-crystal-fiber long-period grating," Opt. Lett., vol. 33, no. 6, pp. 563-565, Mar. 15, 2008.

[19] Z. H. He, Y. N. Zhu, and D. Henry, "Long-period gratings inscribed in air-and water-filled photonic crystal fiber for refractometric sensing of aqueous solution,” Appl. Phys. Lett., vol. 92, no. 4, pp. 044105, Jan. 2008.

[20] S. J. Qiu, et al., "Temperature sensor based on an isopropanol-sealed photonic crystal fiber in-line interferometer with enhanced refractive index sensitivity," Opt. Lett., vol. 37, no. 5, pp. 863-865, Mar. 2012. 